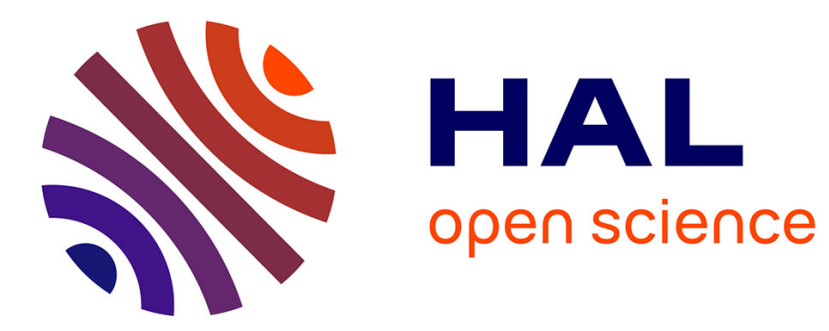

\title{
La cryptosporidiose. Importance de la contamination de l'eau
}

\author{
Muriel Naciri
}

\section{To cite this version:}

Muriel Naciri. La cryptosporidiose. Importance de la contamination de l'eau. Productions Animales, 1992, 5 (5), pp.319-327. hal-00895987

\section{HAL Id: hal-00895987 \\ https://hal.science/hal-00895987}

Submitted on 1 Jan 1992

HAL is a multi-disciplinary open access archive for the deposit and dissemination of scientific research documents, whether they are published or not. The documents may come from teaching and research institutions in France or abroad, or from public or private research centers.
L'archive ouverte pluridisciplinaire HAL, est destinée au dépôt et à la diffusion de documents scientifiques de niveau recherche, publiés ou non, émanant des établissements d'enseignement et de recherche français ou étrangers, des laboratoires publics ou privés. 
INRA Prod. Anim., 1992, 5 (5), 319-327.

\section{Muriel NACIRI}

INRA Station de Pathologie Aviaire et de Parasitologie 37380 Nouzilly

\section{La cryptosporidiose. Importance de la contamination de l'eau}

La Cryptosporidiose est une maladie de découverte récente dont l'agent étiologique est un protozoaire parasite Cryptosporidium. Cette maladie se caractérise, le plus souvent, par des diarrhées néonatales chez les jeunes ruminants et des diarrhées plus ou moins graves chez l'homme selon l'état immunitaire du sujet. En 10 ans, les connaissances concernant Cryptosporidium ont notablement évolué : d'organisme commensal très spécifique d'hôte, il est maintenant reconnu comme ubiquiste, non spécifique et pathogène et la cryptosporidiose est considérée comme une zoonose. Grâce à l'évolution des techniques de mise en évidence et à une meilleure connaissance biologique et épidémiologique du parasite, nous savons à présent qu'il existe une transmission interespèces, mais aussi une contamination d'homme à homme et une contamination par l'eau. Cette dernière voie de transmission, suspectée en 1985, n'est aujourd'hui plus équivoque et pose un grave problème pour la santé.

\section{Résumé}

Cryptosporidium, protozoaire parasite décrit en 1907 par Tyzzer, fut longtemps considéré comme un organisme commensal, rare et spécifique d'hôte. Après 1981, tous les auteurs s'accordèrent à penser que l'espèce C.parvum était commune à tous les mammifères, y compris l'homme, causant des diarrhées néonatales chez les ruminants, des diarrhées à guérison spontanée chez l'homme immunocompétent et des diarrhées cholériformes incurables chez l'homme immunodéficient. La cryptosporidiose était alors considérée comme une zoonose, l'homme s'infectant au contact des animaux malades ou au contact d'animaux de compagnie porteurs sains. Cependant, la cryptosporidiose peut se transmettre directement d'homme à homme comme le démontrent certaines épidémies dans des hôpitaux ou dans des centres aérés, ou indirectement par l'eau de boisson et les aliments souillés. Des recherches effectuées après plusieurs épidémies en Amérique du Nord et au Royaume Uni ont montré que toutes les eaux de surface (lacs, rivières...) sont contaminées par des cryptosporidies. Cette pollution serait principalement due à l'agriculture et à la faune sauvage. Les eaux d'égouts de populations infectées sont aussi source de contamination. Les procédés de traitement des eaux usées, filtration et désinfection, ne sont pas totalement efficaces pour éliminer complètement Cryptosporidium et des oocystes sont retrouvés dans l'eau de boisson. Depuis 1985, les recherches s'intensifient pour mettre au point des techniques fiables afin de déceler la présence des oocystes dans l'eau de boisson, de les identifier et de les inactiver.
Selon Casemore et Jackson (1984) et Casemore (1990), la cryptosporidiose n'est pas nécessairement une zoonose puisqu'il existe une transmission "urbaine" d'homme à homme de la maladie. Il convient donc de définir une zoonose.

La première référence bibliographique aux zoonoses est sans doute biblique et peut être attribuée à Moïse qui avait conscience de l'importance, pour la santé de son peuple, de certaines infections acquises à partir du porc, telles que trichinose et téniasis à Taenia solium, et d'autres types de zoonoses tel que l'Anthrax acquise à partir des cadavres.

Le terme zoonose fut employé par Virchow, il y a plus de 100 ans, pour nommer les maladies transmises des animaux à l'homme. Etymologiquement impropre, il fut souvent très controversé. Les médecins, ayant une vue anthropocentrique, parlèrent de zoonoses pour les maladies transmises de l'animal à l'homme alors que les vétérinaires et biolo- 
gistes employèrent ce terme pour, au contraire, nommer les maladies des animaux pour lesquelles il n'existe pas de maladie humaine avec le même organisme.

En 1951, Kaegel introduit le terme "anthropozoonose" pour inclure toutes les infections que l'homme acquiert des animaux ; mais, en 1957, Wagener l'utilise dans le sens opposé pour décrire les infections des animaux acquises de l'homme.

En 1959, des experts de l'OMS (Organisation Mondiale de la Santé) et de la FAO (Food and Agricultural Organisation) définissent les zoonoses ainsi : "maladies et infections naturellement transmises entre les animaux vertébrés et l'homme". Actuellement le terme "Anthropozoonose" est toujours accepté par ces deux organisations.

En 1989, l'OIE (Office International des Epizooties) a publié un livre sur les zoonoses et maladies transmissibles communes à l'homme et aux animaux, dans lequel 2 types de maladies sont décrits (Acha et Szyfres 1989) :

- les zoonoses strictes, maladies transmises des animaux vertébrés à l'homme, où les animaux jouent un rôle essentiel en entretenant l'infection ou l'infestation dans la nature ; l'homme n'est alors qu'un hôte accidentel ;

- les maladies qui sont communes à l'homme et aux animaux. Dans ce cas l'homme et les animaux contractent l'infection ou sont infestés par les mêmes sources : sol, eau, animaux invertébrés et plantes. Les animaux ne jouent pas, en principe, un rôle essentiel dans le cycle biologique de l'agent causal. Néanmoins, ils peuvent contribuer plus ou moins à sa propagation et à sa transmission effective.

\section{1 / Historique}

Chez des souris n'extériorisant aucun symptôme de maladie, Clarke (1895) observe des petites spores sur l'épithélium gastrique, spores que 12 ans plus tard, Tyzzer (1907) décrira comme C.muris. Puis en 1910-1912, il décrit C.parvum dans l'intestin de ce même animal. Cryptosporidium fut longtemps considéré comme spécifique d'hôte et non pathogène. Seuls, Slavin (1955) décrit C.meleagridis comme responsable de diarrhée dans un élevage de dindons, et Panciera et al (1971) attribuent la diarrhée d'une génisse de 8 mois, aux cryptosporidies. Ces cryptosporidies sont souvent associées à d'autres agents entéropathogènes (rota et corona virus, E.coli K99, Salmonelles..) lors des diarrhées néonatales des jeunes ruminants et il fallut attendre les années 80 pour que leur pouvoir pathogène propre soit reconnu.

Leur présence fut signalée chez l'homme en 1976 : 2 cas, l'un décrit par Meisel et al (1976) chez un patient immunodéprimé, l'autre par Nime et al (1976) chez un enfant immunocompétent, vivant en zone rurale. Mais ce n'est qu'après 1981, avec l'explosion du SIDA, que Cryptosporidium fut reconnu comme agent responsable de diarrhée chez l'homme.
Les recherches s'intensifièrent alors pour trouver un moyen de diagnostic rapide et un traitement, l'infection étant souvent mortelle chez ces sujets.

Jusqu'à ce jour, plus de 100 médicaments ont été testés ; seuls le lasalocid (Göbel 1987) et le lactate d'halofuginone (Naciri et Yvoré 1989) se sont révélés efficaces mais aucun n'est actuellement commercialisé.

Par contre les moyens de diagnostic ont considérablement évolué. Ils ont permis de montrer que Cryptosporidium est également cause de diarrhée chez les sujets immunocompétents, et que l'eau peut être source de contamination.

\section{2 / Cycle de développement du parasite}

Cryptosporidium est un protozoaire parasite appartenant au phylum des Apicomplexa et à la sous-classe des Coccidia. La famille des Cryptosporidiidae renferme un seul genre Cryptosporidium et 4 espèces sont actuellement reconnues (Current et al 1986):

- C.parvum et C.muris chez les mammiferes

- C.baileyi et C.meleagridis chez les oiseaux.

D'autres espèces furent décrites, en particulier chez les reptiles et les poissons, qui n'étaient en réalité que des stades de développement de Sarcocystis.

Cryptosporidium est un parasite monoxène c'est-à-dire à un seul hôte. La forme de résistance et de dissémination de la maladie est l'oocyste, de petite taille (4 à $6 \mu$ ) excrété en grand nombre dans le milieu extérieur avec les fêces des sujets infectés.

Après ingestion, l'oocyste excyste sous l'action de la trypsine et des sels biliaires, libérant ses 4 sporozoïtes ou éléments infectants qui vont se localiser dans la bordure en brosse des cellules épithéliales de l'intestin et préférentiellement dans les entérocytes de l'iléon. Le cycle de développement (figure 1) comporte 2 schizogonies ou multiplications asexuées suivies de la gamogonie. Les schizontes de lère génération matures libèrent 8 mérozoïtes de 1ère génération qui vont réinfecter les cellules voisines donnant naissance à des schizontes I (recyclage) ou à des schizontes de 2ème génération. Les mérozoïtes II (4 par schizonte II) initient la gamogonie. Celle-ci aboutit à la formation de zygotes et d'oocystes. Ces derniers sont émis sporulés dans la lumière intestinale, rejetés avec les fêces dans le milieu extérieur et sont directement infectants pour un autre hôte sensible.

Les particularités de ce cycle consistent dans l'excrétion d'oocystes directement infectants, le recyclage des mérozoïtes de lère génération et la formation d'oocystes à coque fine $(20 \%)$ qui excystent immédiatement, entretenant l'infection. 
Figure 1. Cycle de développement de Cryptosporidium parvum.

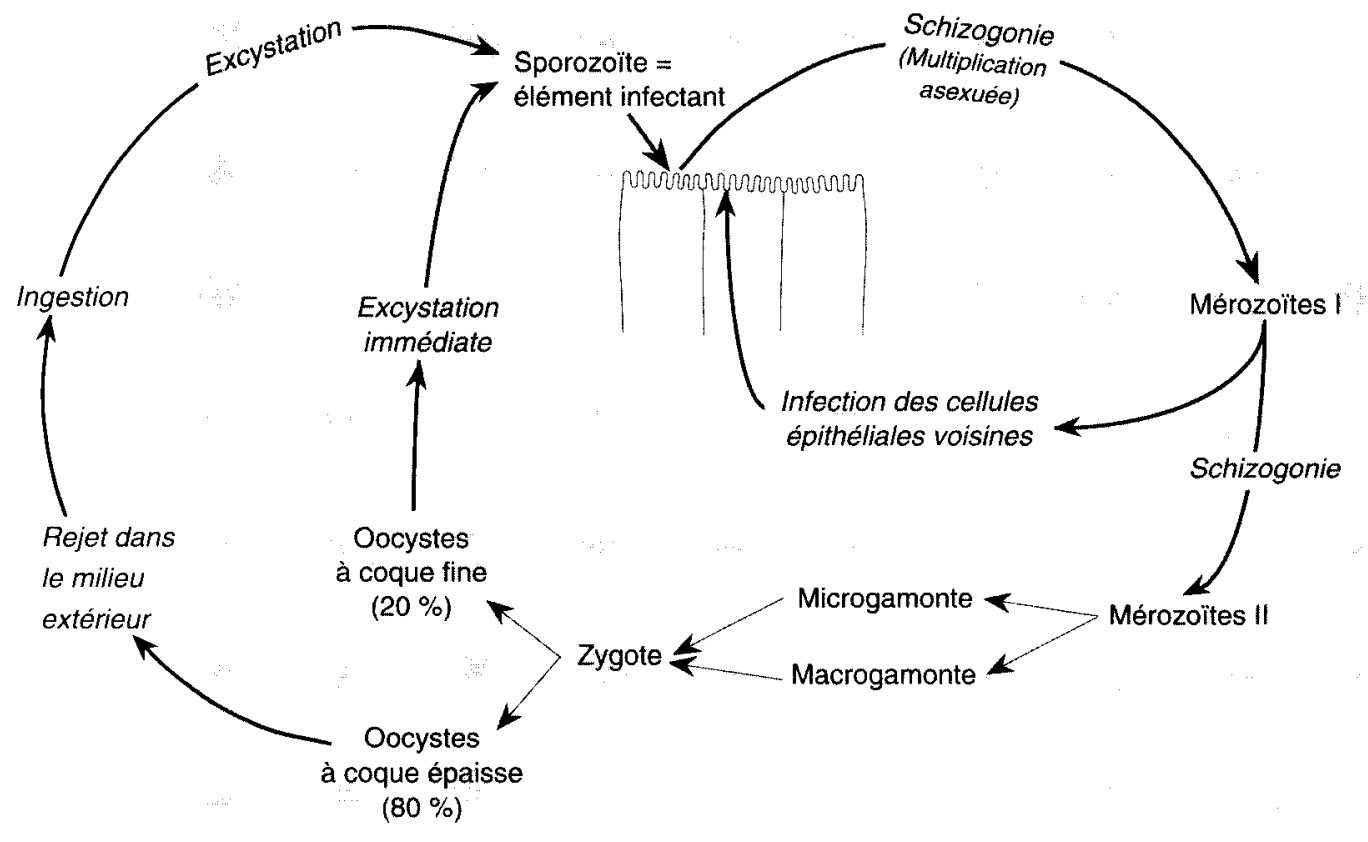

Ces particularités expliqueraient le maintien de l'infection chez les sujets immunodéprimés en l'absence de toute recontamination.

\section{3 / Symptômes chez l'animal et chez l'homme}

C.parvum est l'espèce majeure chez les mammifères, responsable de diarrhées chez les espèces domestiques (bovins, ovins, caprins, équins, porcins...) et chez l'homme. Les parasites affectant les oiseaux semblent difficilement transmissibles à l'homme ou à d'autres mammifères, cependant quelques cas sont cités.

La sensibilité à l'infection est variable chez les animaux:

- diarrhées néonatales graves, souvent mortelles, chez les animaux de rente (bovins, ovins, caprins, porcins..). Après l'âge de 3 semaines ces animaux sont moins sensibles à l'infection par les cryptosporidies.

- absence de symptômes chez les animaux de compagnie : chats, chiens, souris, hamsters, lapins... Ces animaux ne sont pas très sensibles à l'infection par les cryptosporidies, mais peuvent le devenir lors d'immunodépression virale. Porteurs sains ou excréteurs d'un grand nombre de parasites, ils sont une source possible de contamination pour l'homme.

Chez l'homme, la maladie se manifeste sous deux aspects cliniquement distincts :

- gastro-entérite aiguë, à guérison spontanée chez les sujets immunocompétents. La diarrhée, en moyenne 3 litres de selles par jour, dure le plus souvent moins de deux semaines (3 à 6 jours). Elle est accompagnée de nausées, de vomissements, d'anorexie, de douleurs abdominales, de malaises, de pertes de poids et parfois de fièvre. L'excrétion d'oocystes peut durer plusieurs jours après l'arrêt des symptômes.

L'infection à Cryptosporidium permet d'expliquer certaines diarrhées dont l'étiologie était jusque là inconnue, diarrhée dite de retour, diarrhée du voyageur.

- gastro-entérite incurable, chez les sujets immunodéprimés. Les symptômes décrits chez les sujets immunocompétents persistent. L'infection peut s'étendre à d'autres organes que l'intestin, tels que le foie, le pancréas, les poumons. La malabsorption, les vomissements, la diarrhée chronique, cholériforme (17 litres de selles par jour) conduisent à la mort par déshydratation.

\section{4 / Prévalence}

L'importance de la cryptosporidiose n'est pas facile à établir car médecins et vétériencore trop peu de laboratoires recherchent les oocystes dans les selles.

Jusqu'en 1983, les cryptosporidies étaient surtout observées lors d'autopsies de cadavres ou sur biopsies. Les progrès dans les techniques de laboratoire pour lidentification et la numération des oocystes dans les selles ont conduit à un dépistage plus fréquent.

Le parasite a été retrouvé sur tous les continents. La prévalence de la cryptosporidiose chez les sujets immunocompétents est estimée naires ne connaissent pas tous cette maladie et

\section{La contamination se produit par ingestion d'oocystes directement infectants. L'infection est entretenue par une phase de multiplication asexuée et par la production d'oocystes à coque fine, excystant immédiatement.}


à $1-2 \%$ en Europe, $0,6-4,3 \%$ en Amérique du Nord, contre 3-20 \% dans les pays en développement. Cette différence peut sans doute s'expliquer par la mauvaise qualité de l'eau, le manque d'hygiène, les mauvaises conditions d'habitation (concentration des hommes et des animaux dans une même pièce) dans les pays en développement. Dans ces pays, la cryptosporidiose est plus fréquente dans les villes surpeuplées que dans les zones rurales alors qu'il semble que ce soit l'inverse dans les pays industrialisés. La prévalence serait plus élevée pendant les mois les plus chauds et les plus humides (Current et Garcia 1991).

Chez les sujets atteints du SIDA, la prévalence peut aller de 2 à $5 \%$ aux USA en 1985 jusqu'à plus de $50 \%$ en Afrique et Haiti. Ces valeurs sont probablement sous-estimées. En France, selon René et al (1989), 21,2\% des 132 sujets suivis sont infectés par des cryptosporidies. Selon une étude en Grande Bretagne, $19 \%$ des sujets infectés par des cryptosporidies meurent des conséquences de la cryptosporidiose.

Chez les animaux, l'infection est plus commune chez les très jeunes, âgés de moins de 3 semaines (diarrhées néonatales). Chez l'homme, la sensibilité à l'infection dépend plus de l'état immunitaire du sujet, de son état nutritionnel que de son âge. Certaines études menées dans les pays en développement, montrent cependant une plus grande sensibilité et une plus grande fréquence de la cryptosporidiose chez les jeunes enfants de moins de 2 ans (Alpert et al 1984, 1986, Addy et Aikens-Bekoe 1986, Hojlyng et al 1986, Pape et al 1987).

La malnutrition peut contribuer à augmenter la durée de la diarrhée à cryptosporidies et inversement les cryptosporidies peuvent prolonger la diarrhée chez les enfants malnutris. Cryptosporidium serait la cause de diarrhée chronique et l'agent pathogène le plus fréquent chez les enfants de 2 à 15 mois (Sallon et al 1988). La prévalence est augmentée chez les enfants nourris au biberon par rapport à ceux nourris au sein (Mata et al 1984, Pape et al 1987).

Plusieurs hypothèses peuvent être émises pour expliquer cette observation :

- le lait maternel offre peut-être une protection comme cela a pu être montré chez l'animal ;

- le lait du biberon est préparé avec de l'eau contaminée ;

- l'enfant s'amuse avec son biberon, en des endroits qui peuvent être contaminés par des cryptosporidies.

La difficulté pour évaluer la fréquence réelle de ce parasitisme réside également dans le fait que la présence du parasite, aussi bien chez l'homme que chez l'animal, ne s'accompagne pas toujours de diarrhée.

Ungar et al (1988) montrent, par la recherche d'anticorps IgG et IgM, que 19,8\% et $15,5 \%$ de la population du Pérou et du Vénézuela ont eu ou ont une infection crypto- sporidienne. $6 \%$ des individus constituant ces populations sont porteurs d'anticorps. La séroprévalence serait de 25 à $35 \%$ dans les pays industrialisés. Le pouvoir protecteur de ces anticorps est encore très mal connu.

La séroprévalence montre que l'infection cryptosporidienne est plus fréquente que l'on pensait d'après les résultats de recherche des oocystes dans les selles.

\section{5 / Voies de transmission (figure 2)}

La voie de contamination est la voie fécaleorale. Suite aux travaux de Tzipori et al (1980) qui montrèrent, par des expériences de transmissions croisées, la non-spécificité des cryptosporidies, la cryptosporidiose fut considérée comme une zoonose avec transmission directe de l'animal à l'homme. Les animaux de rente et de compagnie furent alors considérés comme réservoirs de parasites et source de contamination pour l'homme. Ceci est probablement vrai, mais n'explique pas les cas de cryptosporidiose observés dans les villes, où le contact avec les animaux est minime. Certains auteurs expliquèrent alors des cas de cryptosporidiose chez des enfants dans des centres aérés, ou chez des personnes hospitalisées, par la transmission d'homme à homme. En 1985, la contamination par l'eau de boisson ou par la nourriture fut suspectée. Pour Jokipii et al (1985), Ma et al (1985) Cryptosporidium est transmis par l'eau de boisson et est responsable de la diarrhée du voyageur.

Avec les progrès réalisés pour rechercher les cryptosporidies dans l'environnement, il apparaît que la transmission zoonotique directe, de l'animal à l'homme, est sans doute de moindre importance par rapport à la contamination par l'eau de boisson, bien qu'animaux sauvages et domestiques soient d'importants réservoirs pour l'infection humaine et la contamination de l'environnement. Matières fécales des animaux et des hommes infectés polluent l'environnement au travers des effluents d'élevage, des épandages sur les sols et des égouts.

\section{6 / Contamination de l'eau}

\section{1 / Techniques de mise en évidence des oocystes dans l'eau}

Afin de déceler de faibles concentrations en oocystes, de grands volumes d'eau sont filtrés (pores $1,2 \mu$ ) puis le résidu est traité en gradient de densité selon la méthode de Musial et al (1987), afin de séparer efficacement les oocystes des débris.

Selon Garcia et al (1987), Sterling et Arrowood (1986), Stibbs et Ongerth (1986), l'emploi des anticorps monoclonaux et polyclonaux, en immunofluorescence directe ou indirecte, a facilité la détection des oocystes :

- Ongerth et Stibbs (1987) ont utilisé un anticorps polyclonal spécifique de la membra- 
Figure 2. Réservoirs et voies de transmission de Crypstosporidium, selon Casemore (1990).

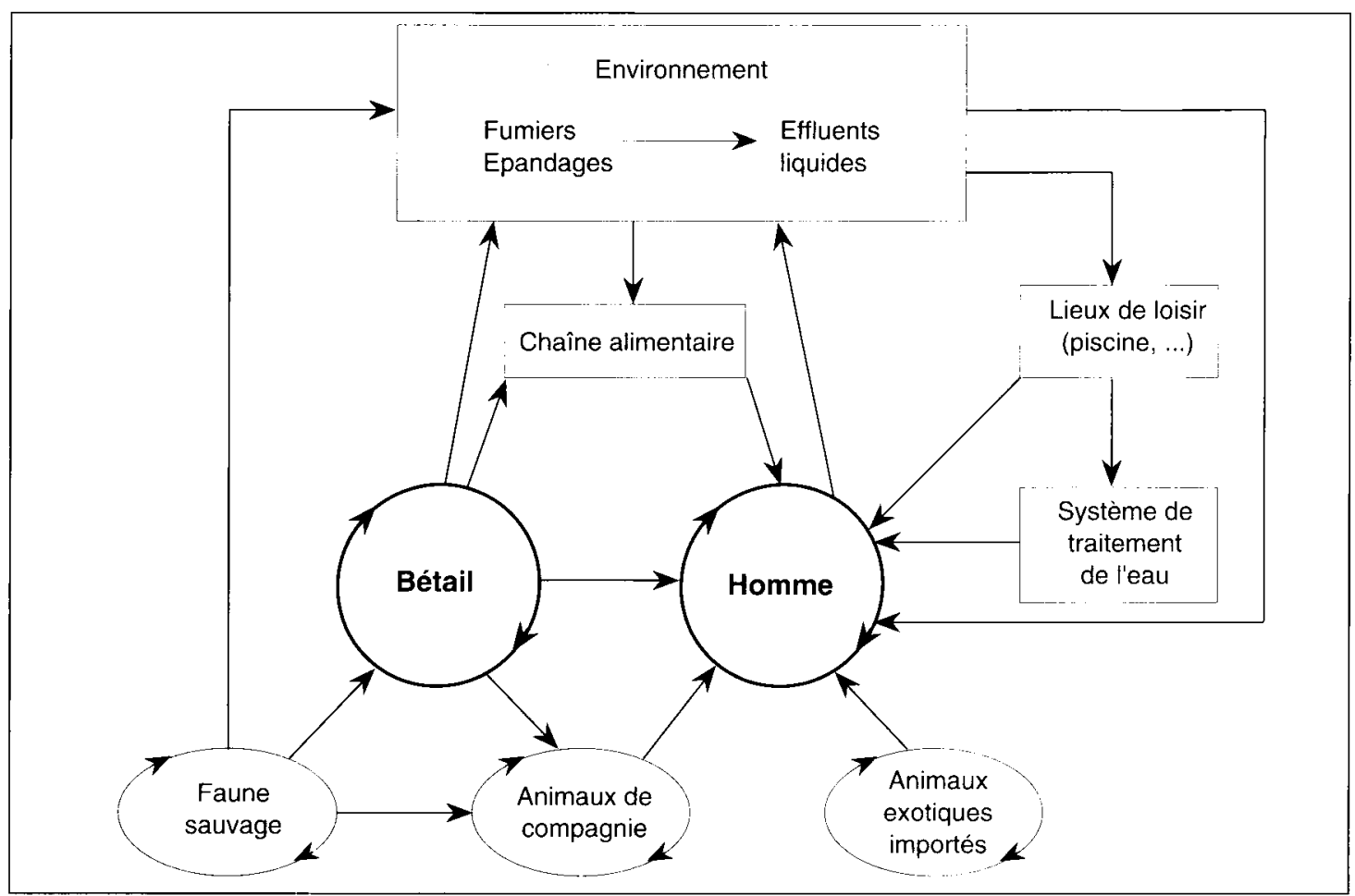

La transmission de l'animal ’̀ l'homme est sans doute moins importante que la contamination par l'eau.

ne du parasite. Cet anticorps permet de détecter les oocystes dans des prélèvements d'humains, de singes, de bovins et ne présente pas de réaction croisée avec les levures (Candida), les Giardia, Entamoeba et Chilomastix. Cependant la spécificité par rapport aux oocystes des oiseaux n'a pas été testée.

- Deux anticorps monoclonaux ont été utilisés dans plusieurs études mais il s'est avéré que l'un croise avec des levures et les oocystes des oiseaux (Sterling et Arrowood 1986) et l'autre, qui ne croise pas avec les levures ni avec C.baileyi, présente quelque affinité pour C.meleagridis (Darlington et Blagburn 1988).

L'utilisation des anticorps monoclonaux présente l'avantage d'une assez grande spécificité puisqu'ils sont générés contre un épitope antigénique spécifique.

L'efficacité des méthodes varie de 9 à $59 \%$ selon la qualité de l'eau. La contamination est donc sans aucun doute sous-estimée. Ongerth et Stibbs (1987) indiquent que des concentrations de 0,44 à 5,6 oocystes/litre peuvent être mathématiquement ajustées à 2 et 112 oocystes/litre. Les échantillons négatifs ne signifient pas nécessairement l'absence d'oocystes contaminants.

La contamination de l'eau est peut-être sous-estimée, mais les méthodes décèlent aussi les oocystes d'oiseaux qui sont sans importance pour la santé de l'homme. La limite la plus importante de ces méthodes réside dans l'incapacité à déterminer la viabilité des oocystes. Le mieux est sans doute de considérer que tout oocyste décelé dans l'eau potable est potentiellement infectieux.

\section{2 / Quelques cas d'infections dues à l'eau}

Les premiers cas de contamination par l'eau aux USA furent rapportés par D'Antonio et al (1985). La diarrhée apparut en juillet 1984 dans une communauté de 1000 personnes. Le taux de diarrhée était 12 fois supérieur à celui des villes avoisinantes. Les cryptosporidies furent retrouvées chez $47 / 79$ sujets avec diarrhée. L'approvisionnement en eau provenait d'un puits artésien dont la javellisation était le seul traitement. Une infiltration par le sol d'eaux d'égouts semblait à l'origine de la contamination de l'eau du puits.

La deuxième épidémie, à Carrollton, en Georgie (USA), en janvier 1987 toucha une région de 64000 personnes ayant le même approvisionnement d'eau (Mason 1988, Hayes et al 1989). Les oocystes furent décelés dans $39 \%$ des selles, en l'absence de tout autre agent entéropathogène. Une enquête révéla que les personnes utilisant l'eau de cette région étaient plus malades que celles utilisant l'eau des puits. La maladie débuta avec $40 \%$ de personnes atteintes et dura plus de 4 semaines. Les oocystes furent décelés dans 7/9 prélèvements d'eau traitée alors que l'eau dans ce comté était soumis au traitement conventionnel, c'est-à-dire, coagulation, sédimentation, filtration rapide sur sable et désinfection par le chlore. D'autres épidémies, tout particulièrement en Grande Bretagne, furent signalées, mettant en cause l'eau de boisson et l'eau des piscines comme source de contamination. 


\section{3 / Niveaux de contamination des eaux}

Un examen des eaux du Royaume Uni et des Etats Unis a montré que les oocystes peuvent être présents dans tous les types d'eaux de surface c'est-à-dire, lacs, réservoirs, fleuves et rivières, avec des concentrations allant de 0,006 à 2,5 oocystes/litre (Packham 1990). Les oocystes sont plus rarement retrouvés dans les eaux de source. La contamination peut généralement être associée à l'agriculture : drainages à partir des cours de fermes, des champs ; épandages de lisiers, écoulements des fumiers ; animaux pâturant près des cours d'eau peuvent aussi augmenter la contamination, sachant que les animaux infectés peuvent excréter jusqu'à $10^{10}$ oocystes par jour pendant 1 à 2 semaines.

Les eaux sales, vidanges et eaux d'égouts provenant de populations infectées sont une autre source de contamination.

Les concentrations d'oocystes dans les eaux usées varient en fonction de la taille de la communauté et de son taux d'infection. Après la fin d'une épidémie, les concentrations dans les eaux usées diminuent de 34 oocystes par litre à 1,2 (Rose et al 1987). Madore et al (1987) rapportent jusqu'à 13700 oocystes par litre avec une moyenne de 5180 oocystes/litre sur 4 échantillons. D'autres études indiquent que le nombre d'oocystes persistant dans les eaux traitées n'est pas négligeable. La dose infectante pour l'homme n'est pas connue, mais Miller et al (1986) affirment que 10 oocystes suffisent pour infecter un singe.

Une enquête réalisée par Rose (1988) dans l'ouest des USA montre que $91 \%$ des eaux d'égout contiennent des cryptosporidies à des taux variables, mais également $77 \%$ des rivières, $75 \%$ des lacs, $83 \%$ des eaux de surface en amont de toute activité humaine, et $28 \%$ des eaux de boisson traitées. Les concentrations d'oocystes dans ces différentes eaux varient et paraissent toujours plus grandes lorsque la pollution agricole semble être la source majeure de contamination.

\section{4 / Interprétation des données}

En 1989, des scientifiques et des spécialistes de l'environnement se sont réunis en Angleterre avec à leur tête Badenoch, spécialiste des gastro-entérites, pour tenter d'expliquer l'épidémie de cryptosporidiose qui a frappé des centaines de personnes dans les régions d'Oxford et de Swindon. Cette épidémie semblait être associée à une source d'eau commune. Le "Public Health Laboratory Service" rapporte qu'en 1989, sur 96111 cas d'infections gastro-intestinales en Angleterre et Pays de Galles, $7904(8,2 \%)$ étaient dus à la cryptosporidiose. En Ecosse, sur 9776 cas, 1273 étaient dus aux cryptosporidies $(12,7 \%)$.

Il ressort des travaux de ce groupe que les statistiques existantes doivent être traitées avec prudence étant donné le nombre limité d'études menées jusqu'à ce jour et qu'il est dif- ficile d'établir la proportion de cas dus à une infection par l'eau, bien qu'elle puisse être envisagée dans les cas où de nombreuses personnes sont affectées dans une aire restreinte.

Tant qu'il n'y a pas de méthodes satisfaisantes pour retrouver les oocystes dans l'environnement, surtout à faible concentration, de méthodes pour les supprimer ou les inactiver, pour différencier les espèces et évaluer leur viabilité, le groupe considère qu'il est difficile de demander un contrôle routinier de l'eau de boisson.

\section{7 / Traitements de l'eau}

Rose (1988) estime qu'une proportion de $28 \%$ des eaux potables contaminées est inacceptable même si le niveau de contamination est faible (0,002-0,009 oocystes/litre)

Le principal traitement des eaux consiste en une filtration suivie d'une désinfection pour inhiber les microorganismes qui n'auraient pu être éliminés par la filtration.

\section{1 / Filtration}

La filtration à elle seule ne parvient pas à éliminer complètement les oocystes du fait de leur petite taille (4 à $5 \mu$ de diamètre) et leur capacité à se déformer. Ils peuvent traverser les membranes de $3 \mu$ voire $1 \mu$. La filtration enlèverait $54,6 \%$ des oocystes des effluents et $91,4 \%$ des oocystes des eaux des rivières.

\section{2 / Désinfection}

Différents désinfectants ont été utilisés et leur efficacité testée en utilisant des modèles animaux (le plus souvent les souris) ou la capacité des oocystes à excyster.

Le formol à $10 \%$, l'acide crésylique à $2,5 \%$ et l'ammoniaque à $5 \%$ seraient des désinfectants efficaces. Par contre, des oocystes incubés à $4^{\circ} \mathrm{C}$ pendant 18 heures dans l'hypochlorite de sodium conservent leur pouvoir infectant. L'hypochlorite de sodium est d'ailleurs utilisé pour aseptiser les oocystes avant les excystations in vitro. Selon Reducker et Speer (1985), Reducker et al (1985) et Woodmansee (1987), l'hypochlorite de sodium facilite l'excystation. Des oocystes prétraités avec 1,05 ou $1,75 \%$ d'hypochlorite excystent facilement à $37^{\circ} \mathrm{C}$ sans utiliser de milieu d'excystation $(0,75 \%$ sels biliaires, $0,25 \%$ de trypsine dans une solution de Hanks). L'hypochlorite de sodium, à $37^{\circ} \mathrm{C}$, altère la perméabilité de la coque de l'oocyste en enlevant la membrane externe et les sporozoïtes s'échappent. Par contre l'excystation est très faible à $4^{\circ} \mathrm{C}$. Alors on peut imaginer une activation du pouvoir infectant des oocystes présents dans les circuits d'eau potable traitée à l'eau de javel. Par contre si l'excystation se poursuit, le désinfectant est efficace car les sporozoïtes ne résistent pas dans l'eau.

Il semble donc que la désinfection avec l'eau de javel (principal procédé de désinfection 
dans de nombreux pays) fournit peu ou pas de protection contre Cryptosporidium. Des concentrations d'eau de javel comprises entre 8000 et $16000 \mathrm{mg} / \mathrm{l}$ sont nécessaires pour tuer les oocystes, or ce niveau de chlore est bien audessus des limites pratiques du traitement de l'eau.

Quelques résultats, sur l'emploi d'autres désinfectants, indiquent l'utilisation du dioxide de chlore, de l'ozone à des concentrations qui pourraient être appliquées dans les procédés du traitement des eaux. L'ozone inactiverait 99\% des oocystes. Les études avec l'ozone doivent être poursuivies concernant la formation de sous-produits biodégradables ou toxiques.

Les cryptosporidies sont 30 fois plus résistantes à l'ozone et 14 fois plus résistantes au dioxyde de chlore que les cystes de Giardia lamblia dans les mêmes conditions (Korich et al 1990)

\section{Conclusion}

Cryptosporidium est un parasite ubiquiste, responsable de diarrhées graves vis-à-vis desquelles il n'existe aucun traitement aussi bien chez l'homme que chez l'animal. Il n'existe pas non plus de système de désinfection efficace de l'environnement. La contamination de l'environnement peut contribuer à maintenir un niveau endémique de la maladie pour la population. Actuellement aucun type de traitement des eaux ne parvient à éliminer complètement les oocystes. Des études sont actuellement en cours dans différents laboratoires ou compagnies des eaux.

Pour conclure nous reprendrons quelques conseils émis lors de la réunion du groupe Badenoch qui, faute de techniques efficaces, ne demande pas de contrôle de routine des eaux mais recommande aux différentes compagnies de développer une stratégie d'action selon les circonstances locales et les risques connus, par exemple dans les cas de :

- pollution agricole exceptionnelle ou pollution par les eaux d'égouts, de l'eau de source ;

- dysfonctionnement du traitement de l'eau ;

- comptages élevés d'organismes indicateurs, ou développement d'une épidémie de cryptosporidiose dans une région.

L'expérience d'épidémies récentes indique que l'examen des filtres (lavage des filtres pour rechercher les cryptosporidies) peut fournir une indication précoce de la contamination de l'eau.

Enfin rappelons, que chez l'homme, la cryptosporidiose peut être bénigne ou dramatique. Nous pensons qu'une recherche systématique du parasite devrait être entreprise dans tous les cas de diarrhées rebelles à tous traitements, leur persistance pouvant déceler un problème d'immunodépression passager ou grave.

\section{Références bibliographiques}

Acha P.N., Szyfres B., 1989. Zoonoses et maladies transmissibles communes à l'homme et aux animaux. O.I.E. 2ème édition Paris, 1063 p.

Addy P.A.K., Aikens-Bekoe P., 1986. Cryptosporidiosis in diarrheal children in Kumasi Ghana. Lancet i:735.

Alpert G., Bell L.M., Kirkpatrick C.E., Budnick L.D., Campos J.M., Friedman H.M., Plotkin S.A., 1984. Cryptosporidiosis in a day-care center. New Engl. J. Med. 311:860-861.

Alpert G., Bell L.M., Kirkpatrick C.E., Budnick L.D., Campos J.M., Friedman H.M., Plotkin S.A., 1986. Outbreak of cryptosporidiosis in a day-care center. Pediatrics 77:152-157.

Casemore D.P., Jackson F.B., 1984. Hypothesis : cryptosporidiosis in human beings is not primarily a zoonosis. J. Infect. 9:153-156.

Casemore D.P., 1990. Epidemiological aspects of human cryptosporidiosis. Epidemiol. Infect. 104:1-28.

Clarke J.J., 1895. A study of coccidia met with in mice. J. Microsc. Soc. 37:277-302.

Current W.L., Upton S.J., Haynes T.B., 1986. The life cycle of Cryptosporidium baileyi n.sp. (Apicomplexa, Cryptosporidiidae) infecting chickens. J. Protozool. 33:289-296.

Current W.L., Garcia L.S,. 1991. Cryptosporidiosis. Clin. Microbiol. Rew. 4:325-358

D'Antonio R.G., Winn R.E., Taylor J.P., Gustafson T.L., Current W.L., Rhodes M.M., Gary G.W., Zajac R.A., 1985. A waterborne outbreak of cryptosporidiosis in normal hosts. Ann. Int. Med. 103:886-888

Darlington M.V., Blagburn B.L., 1988. The merifluorTM immunofluorescent detection procedure is nonspecific for Cryptosporidium species. Ann. Meeting Southeastern Society of Parasitologists Abstract 34, Clemson University, March 30, April 1.

Garcia L.S., Brewer T.C., Boukner D.A., 1987. Fluorescent detection of Cryptosporidium oocysts in human fecal specimens by using monoclonal antibodies. J. Clin. Microbiol. 25:119-121.

Göbel E., 1987. Diagnose und Therapie der akuten Kryptosporidiose beim Kalb. Tierärzlt. Umschau, 42:863-869.

Hayes E.B., Matte T.D., O'Brien T.R., McKinley T.W., Logsdon G.S., Fose J.B., Ungar B.L.P., Word D.M., Pinsky P.F., Cummings M.S., Wilson M.A., Long E.G., Hurwitgs E.S., Juranek D.D., 1989. Large community outbreak of cryptosporidiosis due to contamination of a filtered public water supply. N. Engl. J. Med. 320:1372-1376.

Hojlyng N., Molback K., Jepsen S., 1986. Cryptosporidium spp., a frequent cause of diarrhea in Liberian children. J. Clin. Microbiol. 23:11091113 .

Jokipii L., Pohjola S., Valle S.L., Jokipii A.M., 1985. Cryptosporidiosis associated with traveling and giardiasis. Gastroenterology 89:838-842. 
Kaegel A., 1951. Zoonoses (Anthropozoonoses). Basel : Reinhardt.

Korich D.G., Mead J.R., Madore M.S., Sinclair N.A., Sterling C.R., 1990. Effects of ozone, chlorine dioxyde, chlorine, and monochloramine on Cryptosporidium oocyst viability. Appl. Environ. Microbiol. 56:1423-1428.

Ma P., Kaufman D.L., Helmick C.G., D'Souza A.J., Navin T.R., 1985. Cryptosporidiosis in tourists from the Caribbean. New Engl. J. Med. 312:647-648.

Madore M.S., Rose J.B., Gerba C.P., Arrowood M.J., Sterling C.R., 1987. Occurence of Cryptosporidium oocysts in sewage effluents and selected surface waters. J. Parasitol. 73:702-705.

Mason L., 1988. Experience with Cryptosporidium at Carrollton, Georgia. pp.889-898 in Water Quality Technology Conference. Nov. 15-20, 1987, Baltimore, $\mathrm{MD}$, American Water Works Association, Denver, $\mathrm{CO}$.

Mata L., Bolanos H., Pezarro D., Vives M., 1984 Cryptosporidiosis in children from some highland Costa Rican rural and urban areas. Am. J. Trop. Med. Hyg. 33:24-29.

Meisel J.L., Perera D.R., Meligro C., Rubin C.E. 1976. Overwhelming watery diarrhea associated with Cryptosporidium in an immunosuppressed patient. Gastroenterology 70:1156-1160.

Miller R.A., Brondson M.A., Morton W.R., 1986. Determination of the infectious dose of Cryptosporidium and the influence of inoculum size on disease severity in a primate model. Abstract Annual Meeting of American society of microbiologists, Washington, D.C., 23-28 march p49.

Musial C.E., Arrowood M.J., Sterling C.R., Gerba C.P., 1987. Detection of Cryptosporidium in water by using polypropylene cartridge filters. Appl. Environ. Microbiol. 53:687-692.

Naciri M., Yvoré P., 1989. Efficacité du lactate d'halofuginone dans le traitement de la cryptosporidiose chez l'agneau. Rec. Med. Vet. 165:823-826.

Nime F.A., Burek J.D., Page D.L., Holscher M.A. Yardley J.H., 1976. Acute enterocolitis in a human being infected with the protozoan Cryptosporidium. Gastroenterology 70:592-598.

Ongerth J.E., Stibbs H.H., 1987. Identification of Cryptosporidium oocysts in river water. Appl. Environ. Microbiol. 53:672-676.

Packham R.F., 1990. Cryptosporidium and water supplies - the Badenoch report. J. I.W.E.M. 4:578 580 .

Panciera R.J., Thomassen R.W., Gardner F.M., 1971. Cryptosporidial infection in a calf. Vet. Pathol. 8:479-484

Pape J.W., Levine E., Beaulieu M.E., Marshall F., Verdier R., Johnson W.D., 1987. Cryptosporidiosis in Haitian children. Am. J. Trop. Med. Hyg. 36:333337.

Reducker D.W., Speer C.A., 1985. Factors influencing excystation in Cryptosporidium oocysts from cattle. J. Parasitol. 71:112-115.

Reducker D.W., Speer C.A., Blixt J.A., 1985. Ultrastructural changes in the oocyst wall during excystation of Cryptosporidium parvum (Apicomplexa ; Eucoccidiorida). Can. J. Zool. 63:1892-1896.
René E., Marche C., Regnier B., Saimot A.G., Vilde J.L., Perrone C., Michon C., Wolf M., Chevalier T., Villot T., 1989. Intestinal infections in patients with acquired immunodeficiency syndrome. A prospective study in 132 patients. Dig. Dis. Sci. 34:773-780.

Rose J.B., Gerba C.P., Logsdon G.A., Hayes E., Matti T., Juranek D.D., 1987. Occurence of Cryptosporidium oocysts in a distribution system after a waterborne outbreak. Water Quality Technology Conference. Baltimore, MD. Nov. 15-19. J. Am. Water Works Assoc. 79:47.

Rose J.B., 1988. Occurence and significance of Cryptosporidium in water. J. Am. Water Works Assoc. 80:53-58.

Rose J.B., Kayed D., Madore M.S., Gerba C.P., Arrowood M.J., Sterling C.R., 1988. Methods for the recovery of Giardia and Cryptosporidium from environmental waters and their comparative occurence. Advances in Giardia Research, Wallis P, and Hammond B (eds) Univ. Calgary Press p 205209 .

Rose J.B., 1990. Occurence and control of Cryptosporidium in drinking water. Gordon A. McFeters Ed, Drinking Water Microbiology p 293321.

Sallon S., Deckelbaum R.J., Schmid I.I., Harlap S. Baras M., Spira D.T., 1988. Cryptosporidium, malnutrition, and chronic diarrhea in children. Am. J. Dis. Child. 142:312-315.

Slavin D., 1955. Cryptosporidium meleagridis (sp. nov.). J. Comp. Pathol. 65:262-266

Sterling C.R., Arrowood M.J., 1986. Detection of Cryptosporidium sp. infections using a direct immunofluorescent assay. Pediatr. Infect. Dis. 5:S139-S142.

Stibbs H.H., Ongerth J.E., 1986. Immunofluorescence detection of Cryptosporidium oocysts in fecal smears. J. Clin. Microbiol. 24:517-521.

Tyzzer E.E., 1907. A sporozoan found in the peptic glands of the common mouse. Proc. Soc. Exp. Biol. Med. 5:12-13

Tyzzer E.E., 1910. An extracellular coccidium, Cryptosporidium muris (gen. et $\mathrm{sp}$. nov.) of the gastric glands of the common mouse. J. Med. Res. 23:487-516.

Tyzzer E.E., 1912. Cryptosporidium parvum (sp. nov.) a coccidium found in the small intestine of the common mouse. Arch. Protistenkd. 26:394-412

Tzipori S., Angus K.W., Campbell I., Gray E.W., 1980. Cryptosporidium : evidence for a single-species genus. Infect. Immun. 30:884-886.

Ungar B.L.P., Gilman R.H., Lanata C.F., PerezSchael I., 1988 . Seroepidemiology of Cryptos poridium infection in two Latin American populations. J. Inf. Dis. 57:551-556.

Wagener K., 1957. Zoonoses. Berl. Munch. Tierarztl. Wochenschr. 70:12

Woodmansee D.B., 1987. Studies of in vitro excystation of Cryptosporidium parvum from calves. J. Protozool. 34:398-402. 


\section{Summary}

Crytposporidiosis. Importance of water as a source of infection.

Described in 1907, Cryptosporidium, a protozoan parasite, was considered as a rare, commensal and specific organism. After 1981, C.parvum was recognized as a common source of contamination in all mammals including humans. Symptoms are neonatal diarrhea in ruminants and self limiting or life threatening diarrhea in immunocompetent or immunodeficient hosts, respectively. The first reports indicated that infected animals were the source of human infections and that cryptosporidiosis was a zoonotic disease. Nevertheless, cryptosporidiosis may be transmitted directly from person to person and indirectly by water. Pollution of all types of surface water, lakes, reservoirs, rivers etc can result from agriculture but sewage from infected populations are also a source of contamination. The sewage treatment pro. cesses, filtration and desinfection, are not fully effective and oocysts have been found in drinking water in North America and the United Kingdom. From 1985, studies have been carried out to enumerate, identify and inactivate Cryptosporidium oocysts in water.

NACIRI Muriel, 1992. La cryptosporidiose. Importance de la contamination de l'eau. INRA Prod. Anim., 5 (5), 319- 327 